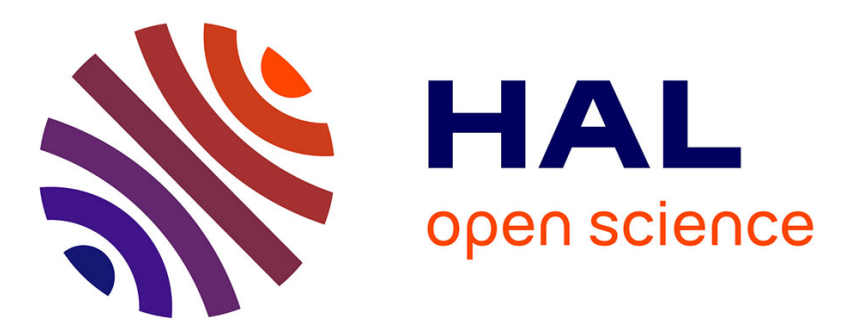

\title{
À propos du rôle (dé)stabilisateur de l'hétérogénéité $<$ br / > dans les économies à générations imbriquées
}

Stefano Bosi, Thomas Seegmuller

\section{To cite this version:}

Stefano Bosi, Thomas Seegmuller. À propos du rôle (dé)stabilisateur de l'hétérogénéité<br />dans les économies à générations imbriquées. Revue Economique, 2007, 58 (3), pp.629-636. $10.3917 /$ reco.583.0629 . halshs-00194250

\section{HAL Id: halshs-00194250 \\ https://shs.hal.science/halshs-00194250}

Submitted on 6 Dec 2007

HAL is a multi-disciplinary open access archive for the deposit and dissemination of scientific research documents, whether they are published or not. The documents may come from teaching and research institutions in France or abroad, or from public or private research centers.
L'archive ouverte pluridisciplinaire HAL, est destinée au dépôt et à la diffusion de documents scientifiques de niveau recherche, publiés ou non, émanant des établissements d'enseignement et de recherche français ou étrangers, des laboratoires publics ou privés. 


\title{
A propos du rôle (dé)stabilisateur de l'hétérogénéité dans les économies à générations imbriquées*
}

\author{
Stefano Bosi ${ }^{\dagger}$ et Thomas Seegmuller ${ }^{\ddagger}$
}

\begin{abstract}
Un grand nombre de travaux ont cherché à exhiber les conditions sous lesquelles des fluctuations dues à la volatilité des anticipations des agents et des cycles endogènes apparaissent, mais peu d'entre eux se sont intéressés au rôle de l'hétérogénéité des consommateurs. Dans cet article, nous abordons la question dans un modèle à générations imbriquées avec offre de travail endogène. En considérant une variation de l'hétérogénéité à moyenne constante, nous montrons qu'une plus grande ou plus faible dispersion n'affecte nullement la dynamique locale. L'agent représentatif est donc une bonne représentation d'une population hétérogène.
\end{abstract}

\section{ON THE (DE)STABILIZING ROLE OF HETEROGENEITY IN OVERLAPPING GENERATIONS ECONOMIES}

\begin{abstract}
A large literature has tried to find conditions such that expectationsdriven fluctuations and endogenous cycles appear, but only few articles have focused on the role of consumers' heterogeneity. In our paper, we address this issue by considering an overlapping generations economy with endogenous labor supply. Introducing a variation of heterogeneity, while keeping the mean as constant, we show that a greater or a weaker dispersion does not affect the local dynamics. Therefore, in our framework, the representative agent is a good representation of an heterogeneous population.
\end{abstract}

Classification JEL : C62, E32

\footnotetext{
*Nous remercions Francesco Magris pour ses commentaires et ses suggestions. Toutes les erreurs subsistantes sont de notre reponsabilité.

†EQUIPPE, Département d'économie, Université de Lille 1, Bât. SH2, Bureau 132, Cité Scientifique, 59655 Villeneuve d'Ascq cedex, France. Tel : (33) 3204370 12. EPEE, Université d'Evry. E-mail : stefano.bosi@univ-lille1.fr.

${ }^{\ddagger}$ CNRS et Centre d’Economie de la Sorbonne, Paris School of Economics, Université Paris 1, 106-112, bd de l'Hôpital, 75647 Paris Cedex 13, France. Tel : (33) 14407 81 99, Fax : (33) 1440782 31. E-mail : seegmu@univ-paris1.fr.
} 


\section{INTRODUCTION}

Dans les deux dernières décennies, un grand nombre de travaux ont exploité l'indétermination de l'équilibre et des non-linéarités suffisamment fortes pour montrer l'existence de fluctuations dues à la volatilité des anticipations et de cycles endogènes. ${ }^{1}$ La plupart de ces contributions ont supposé que le comportement des consommateurs se résume à celui d'un agent représentatif et peu d'entre elles se sont intéressées au rôle de l'hétérogénéité des agents sur l'apparition des fluctuations économiques. ${ }^{2}$ En outre, parmi ces quelques travaux qui ont analysés de quelle manière l'hétérogénéité affecte les fluctuations, ceux qui se sont intéressés au lien entre consommateurs hétérogènes et indétermination de l'équilibre (Ghiglino et Sorger [2002], Ghiglino et OlszakDuquenne [2005]) se sont surtout focalisés sur l'hétérogénéité de la richesse initiale ou ont peu analysé le rôle du marché du travail, bien qu'il est connu que celui-ci est prépondérant dans l'apparition de fluctuations dues à la volatilité des anticipations.

Partant de ce constat, nous étudions dans cet article le rôle de l'hétérogénéité des consommateurs sur l'indétermination en centrant notre analyse sur la diversité des préférences et le marché du travail. Nous choisissons comme cadre d'analyse un modèle à générations imbriquées simple dans lequel les consommateurs, vivant deux périodes, offrent du travail à la première période de leur vie, épargnent sous forme de capital et consomment uniquement à la deuxième période de leur vie (Reichlin [1986]). Au lieu qu'un agent représentatif représente chaque génération, nous supposons que la population de celle-ci se compose de consommateurs hétérogènes. Dans ce modèle, le comportement de chaque agent peut se résumer à son offre de travail, comme fonction du salaire réel et du taux d'intérêt réel futur anticipé. Pour exhiber les conditions d'indétermination de l'équilibre, nous analysons la stabilité locale de l'état stationnaire. Le paramètre qui représente le comportement des consommateurs est alors l'élasticité de l'offre de travail. Accroître l'hétérogénéité des consommateurs revient à augmenter la dispersion de ces élasticités tout en supposant leur moyenne comme constante. En utilisant cette mesure de l'hétérogénéité, nous montrons que toute modification de la dispersion de la population n'affecte pas la dynamique locale, c'est-à-dire l'apparition de fluctuations dues à la volatilité des anticipations et de cycles endogènes. Une population plus hétérogène ne favorise ni ne stabilise l'apparition de fluctuations endogènes. Dans ce type de modèle, l'agent représentatif semble donc être une bonne approximation de l'hétérogénéité des comportements, même si au contraire du modèle à agent à durée de vie infinie, les théorèmes du bien-être ne s'appliquent pas.

Dans la suite de ce travail, nous allons d'abord présenter le modèle et définir l'équilibre intertemporel. Dans la troisième section, nous établissons l'existence

\footnotetext{
${ }^{1}$ Pour une revue de la littérature, on peut se référer à Guesnerie et Woodford [1992] ou Benhabib et Farmer [1999].

${ }^{2}$ Il faut cependant faire exception des travaux qui ont introduit de l'hétérogénéité, en particulier dans le degré d'impatience des agents, pour exploiter l'existence de contraintes de liquidité ou d'endettement. Voir par exemple Woodford [1986] ou Becker et Foias [1987, 1994].
} 
d'un état stationnaire. Enfin, dans la dernière section, nous analysons la dynamique locale et étudions l'influence de l'hétérogénéité sur la stabilité de l'état stationnaire.

\section{LE MODĖLE}

Nous considérons une économie parfaitement concurrentielle en temps discret, $t=1,2, \ldots,+\infty$. Dans la suite de la section, nous allons d'abord présenter le comportement des producteurs, puis celui des consommateurs hétérogènes et allons conclure par la définition de l'équilibre intertemporel.

\section{Les producteurs}

Au contraire des consommateurs, le secteur de la production est parfaitement homogène. Aussi allons-nous supposer que le bien final, qui sert de numéraire, est produit par une firme représentative à partir de deux facteurs de production, le capital $k_{t-1}$ et le travail $l_{t}$, et de la technologie à rendements constants :

$$
y_{t}=A f\left(a_{t}\right) l_{t},
$$

où $A>0$ est un paramètre, $a_{t} \equiv k_{t-1} / l_{t}$ le ratio capital-travail et $f(a)$ la fonction de production intensive qui satisfait l'hypothèse :

HYPOTHĖSE 1. La fonction $f(a)$ est continue pour tout $a \geq 0$, prend des valeurs positives et est continuement différentiable, autant de fois que cela est nécessaire, pour tout $a>0$, avec $f^{\prime}(a)>0>f^{\prime \prime}(a)$.

En notant $w_{t}$ le salaire réel et $r_{t}$ le taux d'intérêt réel, les profits de la firme représentative s'écrivent $y_{t}-w_{t} l_{t}-r_{t} k_{t-1}$. La maximisation de ces profits conduit aux deux expressions :

$$
r_{t}=A \rho\left(a_{t}\right) \text { et } w_{t}=A \omega\left(a_{t}\right),
$$

où $\rho(a) \equiv f^{\prime}(a)$ et $\omega(a) \equiv f(a)-a f^{\prime}(a)$. Avant de présenter le comportement des consommateurs, nous définissons $s(a) \equiv a f^{\prime}(a) / f(a) \in(0,1)$ comme étant la part du capital dans la production. Par ailleurs, l'élasticité de substitution capital-travail $\sigma(a)$ satisfait l'égalité $1 / \sigma(a)=a \omega^{\prime}(a) / \omega(a)-a \rho^{\prime}(a) / \rho(a)$. En utilisant $\omega^{\prime}(a)=-a \rho^{\prime}(a)$, on obtient finalement les deux élasticités suivantes :

$$
\begin{aligned}
a \rho^{\prime}(a) / \rho(a) & =-[1-s(a)] / \sigma(a), \\
a \omega^{\prime}(a) / \omega(a) & =s(a) / \sigma(a) .
\end{aligned}
$$




\section{Les consommateurs}

Les consommateurs sont en générations imbriquées. La population totale est constante et la taille de chaque génération normalisée à 1. Les agents vivent deux périodes, offrent leur travail lorsqu'ils sont jeunes, épargnent sous forme de capital, et consomment uniquement lorsqu'ils sont vieux. Au lieu de supposer que le comportement de chaque génération se résume à celui d'un agent représentatif, chaque génération est composée de $n$ types de consommateurs $i(i=1,2, \ldots, n)$, qui sont en proportion constante $\alpha_{i} \in[0,1]$, avec $\sum_{i=1}^{n} \alpha_{i}=1$. Leur hétérogénéité se traduit par des dotations initiales en travail et des préférences différentes. Celles-ci sont séparables entre consommation future et loisir et, pour l'agent de type $i$, sont représentées par la fonction d'utilité:

$$
U_{i}\left(c_{i t+1} / B_{i}\right)-V_{i}\left(l_{i t}\right),
$$

où $l_{i t}$ représente l'offre de travail d'un consommateur de type $i, c_{i t+1}$ sa consommation future, et $B_{i}>0$ un paramètre. Par ailleurs, les fonctions $U_{i}\left(x_{i}\right)$ et $V_{i}\left(l_{i}\right)$ satisfont l'hypothèse $:^{3}$

HYPOTHĖSE 2. Les fonctions $U_{i}\left(x_{i}\right)$ et $V_{i}\left(l_{i}\right)$ sont continues pour tout $x_{i} \geq 0$ et $0 \leq l_{i} \leq l_{i}^{*}$, où $l_{i}^{*}$ est la dotation en travail. Elles admettent des dérivées continues de tout ordre requis, pour tout $x_{i}>0$ et $0<l_{i}<l_{i}^{*}$, avec $U_{i}^{\prime}\left(x_{i}\right)>$ $0>U_{i}^{\prime \prime}\left(x_{i}\right), V_{i}^{\prime}\left(l_{i}\right)>0, V_{i}^{\prime \prime}\left(l_{i}\right) \geq 0$ et $-U_{i}^{\prime \prime}\left(x_{i}\right) x_{i} / U_{i}^{\prime}\left(x_{i}\right)<1$. Par ailleurs, $\lim _{l_{i} \rightarrow l_{i}^{*}} V_{i}^{\prime}\left(l_{i}\right)=+\infty$.

Le travail offert par chaque consommateur est rémunéré au salaire concurrentiel $w_{t}$. Par ailleurs, chaque agent utilise son épargne pour acheter du capital et transférer ainsi du pouvoir d'achat en vue de sa consommation future. Un agent de type $i$ maximise donc son utilité (4) sous les deux contraintes budgétaires :

$$
\begin{aligned}
k_{i t} & =w_{t} l_{i t}, \\
c_{i t+1} & =R_{t+1} k_{i t} .
\end{aligned}
$$

où $R_{t+1} \equiv 1-\delta+r_{t+1}$ représente le facteur d'intérêt réel et $\delta \in(0,1)$ le taux de dépréciation du capital. On peut en déduire la condition du premier ordre du programme de maximisation des consommateurs :

$$
u_{i}\left(c_{i t+1} / B_{i}\right)=v\left(l_{i t}\right),
$$

où $u_{i}\left(x_{i}\right) \equiv x_{i} U^{\prime}\left(x_{i}\right)$ et $v_{i}\left(l_{i}\right) \equiv l_{i} V^{\prime}\left(l_{i}\right) .{ }^{4}$ En utilisant les équations (5) et (6), l'égalité $(7)$ définit implicitement l'offre de travail $l_{i t}$ comme une fonction du salaire réel $w_{t}$ et du facteur d'intérêt réel futur $R_{t+1}$ :

$$
l_{i t}=\lambda_{i}\left(z_{i t}\right), \text { avec } z_{i t} \equiv R_{t+1} w_{t} / B_{i} .
$$

\footnotetext{
${ }^{3}$ Pour simplifier l'écriture, nous utilisons la notation $x_{i} \equiv c_{i} / B_{i}$.

${ }^{4} \mathrm{~A}$ la première période $(t=1)$, il y a une génération de $n$ types de vieux qui vivent une seule période et consomment leurs dotations initiales (hétérogènes) en capital $k_{i 0}>0$.
} 
Il faut remarquer que les différentes fonctions $\lambda_{i}\left(z_{i}\right)$, qui résultent du choix intertemporel des consommateurs entre loisir et consommation future, déterminent le comportement hétérogène des différents types d'agents. ${ }^{5}$ Avant de déterminer l'équilibre intertemporel, nous définissons l'élasticité de l'offre de travail d'un consommateur de type $i$. En utilisant les équations (5), (6) et (7), nous obtenons :

$$
\varepsilon_{\lambda_{i}}\left(z_{i}\right) \equiv \frac{\lambda_{i}^{\prime}\left(z_{i}\right) z_{i}}{\lambda_{i}\left(z_{i}\right)}=\frac{1-\varepsilon_{u_{i}}\left(x_{i}\right)}{\varepsilon_{v_{i}}\left(l_{i}\right)+\varepsilon_{u_{i}}\left(x_{i}\right)} \geq 0,
$$

où on note $\varepsilon_{u_{i}}\left(x_{i}\right) \equiv-U_{i}^{\prime \prime}\left(x_{i}\right) x_{i} / U_{i}^{\prime}\left(x_{i}\right) \in(0,1)$ et $\varepsilon_{v_{i}}\left(l_{i}\right) \equiv V_{i}^{\prime \prime}\left(l_{i}\right) l_{i} / V_{i}^{\prime}\left(l_{i}\right) \geq$ 0 .

\section{L'équilibre intertemporel}

L'équilibre sur le marché du capital se traduit par $k_{t}=\sum_{i=1}^{n} \alpha_{i} k_{i t}$, celui sur le marché du travail par $l_{t}=\sum_{i=1}^{n} \alpha_{i} l_{i t}{ }^{6}$ En utilisant ces deux égalités et l'équation (5), on obtient :

$$
k_{t}=w_{t} l_{t} .
$$

Par ailleurs, en substituant (8) dans l'équation définissant l'équilibre sur le marché du travail, on déduit :

$$
l_{t}=\sum_{i=1}^{n} \alpha_{i} \lambda_{i}\left(R_{t+1} w_{t} / B_{i}\right) .
$$

En substituant les expressions du salaire réel et du taux d'intérêt réel (équations (1)) dans (9) et (10), nous pouvons définir un équilibre intertemporel :

PROPOSITION 1. Un équilibre intertemporel est une suite $\left\{k_{t-1}, l_{t}\right\}_{t=1}^{\infty}$ telle que :

$$
\begin{aligned}
k_{t} & =A \omega\left(k_{t-1} / l_{t}\right) l_{t}, \\
l_{t} & =\sum_{i=1}^{n} \alpha_{i} \lambda_{i}\left[R\left(k_{t} / l_{t+1}\right) A \omega\left(k_{t-1} / l_{t}\right) / B_{i}\right],
\end{aligned}
$$

ò̀ $R\left(k_{t} / l_{t+1}\right)=1-\delta+A \rho\left(k_{t} / l_{t+1}\right)$.

Notons que la dynamique de l'économie est définie par les équations (11) et (12), c'est-à-dire par un système dynamique à deux dimensions avec une variable prédéterminée, le capital. ${ }^{7}$

\footnotetext{
${ }^{5} \mathrm{Il}$ faut noter qu'on tient compte de l'hétérogénéité des dotations en travail à travers les désutilités du travail $V_{i}\left(l_{i}\right)$, et donc à travers les offres de travail $l_{i t}=\lambda_{i}\left(z_{i t}\right)$.

${ }^{6}$ Evidemment, si ces deux égalités sont satisfaites, le marché du bien final est aussi équilibré, par la loi de Walras.

${ }^{7}$ On peut remarquer que les dotations initiales en capital $k_{i 0}$ reçues par la première génération d'agents, qui ne vivent qu'une seule période, n'influencent pas la dynamique.
} 


\section{EXISTENCE DE L'ETAT STATIONNAIRE}

Un état stationnaire du système dynamique (11)-(12) peut se définir comme étant une solution $\left(k, l, l_{1}, \ldots, l_{n}\right)$ qui satisfait les équations :

$$
\begin{aligned}
k & =A \omega(k / l) l, \\
l_{i} & =\lambda_{i}\left(\left[1-\delta+A \rho(k / l) A \omega(k / l) / B_{i}\right), \text { pour } i=1, \ldots, n,\right. \\
l & =\sum_{i=1}^{n} \alpha_{i} l_{i} .
\end{aligned}
$$

Dans cette section, nous allons établir l'existence d'un état stationnaire en suivant l'analyse proposée par Cazzavillan et al. [1998]. ${ }^{8}$ A cet effet, nous allons choisir de manière appropriée les paramètres $A, B_{1}, \ldots, B_{n}>0$ pour que $\left(k, l_{1}, \ldots, l_{n}\right)=(1,1, \ldots, 1)$ soit une solution stationnaire. ${ }^{9}$ A partir de l'équation (13), il existe une unique solution $A>0$ telle que :

$$
A=1 / \omega(1) .
$$

En utilisant la définition de $\lambda_{i}$, on peut réécrire (14), évaluée en $\left(k, l_{1}, \ldots, l_{n}\right)$ $=(1,1, \ldots, 1)$, de la manière suivante :

$$
u_{i}\left([1-\delta+A \rho(1)] A \omega(1) / B_{i}\right)=v(1), \text { pour } i=1, \ldots, n .
$$

Sous l'Hypothèse 2, les fonctions $u_{i}\left(x_{i}\right)$ sont croissantes. Par conséquent, si la condition :

$$
\lim _{x_{i} \rightarrow 0} u_{i}\left(x_{i}\right)<v(1)<\lim _{x_{i} \rightarrow+\infty} u_{i}\left(x_{i}\right), \text { pour } i=1, \ldots, n .
$$

est respectée, des solutions $B_{1}, \ldots, B_{n}>0$, satisfaisant (17) pour tout $i=$ $1, \ldots, n$, existent. Remarquons finalement que l'équation (15) est également respectée puisque $\sum_{i=1}^{n} \alpha_{i}=1$.

Ce résultat, portant sur l'existence d'un état stationnaire, peut s'énoncer :

PROPOSITION 2. Sous les Hypothèses 1 et 2, si l'inégalité (18) est assurée, $\left(k, l, l_{1}, \ldots, l_{n}\right)=(1,1,1, \ldots, 1)$ est un état stationnaire du système dynamique (11)-(12) si et seulement si $A, B_{1}, \ldots, B_{n}$ satisfont les équations (16) et (17).

\section{DYNAMIQUE LOCALE}

Dans cette section, nous étudions de quelle manière la prise en compte de consommateurs hétérogènes influe sur les propriétés de stabilité de l'état

\footnotetext{
${ }^{8}$ Par souci de concision, nous ne discuterons pas du nombre d'états stationnaires, puisque nous nous focaliserons sur la dynamique locale dans le voisinage de l'état stationnaire dont nous établissons l'existence.

${ }^{9}$ Evidemment, cette solution stationnaire est également caractérisée par $l=1$.
} 
stationnaire. Comme nous l'avons souligné précédemment, le comportement hétérogène des consommateurs est représenté par la fonction $\lambda_{i}\left(z_{i}\right)$. Par conséquent, l'hétérogénéité des agents affecte la dynamique locale à travers les élasticités de cette fonction, notées $\varepsilon_{\lambda_{i}}$ lorsqu'elles sont évaluées à l'état stationnaire. Dans la suite, nous nous intéressons à des variations de l'hétérogénéité qui modifient la dispersion des élasticités $\varepsilon_{\lambda_{i}}$ tout en gardant leur moyenne $\bar{\varepsilon}_{\lambda} \equiv \sum_{i=1}^{n} \alpha_{i} \varepsilon_{\lambda_{i}}$ constante.

Pour effectuer cette analyse, nous allons commencer par différencier le système dynamique (11)-(12) dans le voisinage de l'état stationnaire $\left(k, l, l_{1}, \ldots, l_{n}\right)$ $=(1,1,1, \ldots, 1)$. En notant $s \equiv s(1), \sigma \equiv \sigma(1)$ et en utilisant (2), (3) et l'égalité $A \rho(1)=s /(1-s)$, nous obtenons :

$$
\begin{aligned}
\frac{d k_{t}}{k}= & \frac{s}{\sigma} \frac{d k_{t-1}}{k}+\left(1-\frac{s}{\sigma}\right) \frac{d l_{t}}{l} \\
\frac{d l_{t+1}}{l}= & \left(\frac{s}{\sigma}+\delta-\frac{1}{1-s}\right) \frac{d k_{t-1}}{k} \\
& +\left[1-\frac{s}{\sigma}+\left(\frac{1}{1-s}-\delta\right)\left(\frac{\sigma}{s \bar{\varepsilon}_{\lambda}}+1\right)\right] \frac{d l_{t}}{l} .
\end{aligned}
$$

Nous en déduisons les expressions de la trace $T$ et du déterminant $D$ de la matrice Jacobienne associée, qui représentent respectivement la somme et le produit des valeurs propres, solutions du polynôme caractéristique $P(\mu) \equiv$ $\mu^{2}-T \mu+D=0$ :

$$
\begin{aligned}
T & =1+\frac{1}{1-s}-\delta+\frac{\sigma}{s}\left(\frac{1}{1-s}-\delta\right) \frac{1}{\bar{\varepsilon}_{\lambda}} \\
D & =\left(\frac{1}{1-s}-\delta\right)\left(1+\frac{1}{\bar{\varepsilon}_{\lambda}}\right) .
\end{aligned}
$$

Pour étudier les propriétés de stabilité locale, nous faisons l'hypothèse :

HYPOTHĖSE $3 . s<\delta /(1+\delta)$.

Comme dans le modèle à générations imbriquées où les agents vivent deux périodes, le taux de dépréciation du capital est généralement supposé proche de 1 , cette hypothèse n'est pas très restrictive pour la part du capital dans la production $s$.

En utilisant cette dernière hypothèse, nous déduisons le résultat suivant :

PROPOSITION 3. Soit $\bar{\varepsilon}_{\lambda_{H}} \equiv[1-\delta(1-s)] /[\delta-s(1+\delta)]$. Si les Hypothèses 1 - 3 sont vérifiées, on a:

(i) lorsque $\sigma>s$, l'équilibre stationnaire est un point-selle;

(ii) lorsque $\sigma<s$, l'équilibre stationnaire est localement stable (indéterminé) pour $\bar{\varepsilon}_{\lambda}>\bar{\varepsilon}_{\lambda_{H}}$, une bifurcation de Hopf apparaît génériquement pour $\bar{\varepsilon}_{\lambda}=\bar{\varepsilon}_{\lambda_{H}}$, et l'équilibre stationnaire est instable pour $\bar{\varepsilon}_{\lambda}<\bar{\varepsilon}_{\lambda_{H}}$. 
Preuve. La dynamique locale se déduit de l'étude de $T, D$ et du polynôme caractéristique $P(\mu)$. Tout d'abord, comme $T>0$ et $D>0$, on a $P(-1)=$ $1+T+D>0$. Par ailleurs, en utilisant (19) et (20), on obtient :

$$
P(1)=1-T+D=\frac{1}{\bar{\varepsilon}_{\lambda}}\left(\frac{1}{1-s}-\delta\right)\left(1-\frac{\sigma}{s}\right) .
$$

Par conséquent, si $\sigma>s, P(1)$ est strictement négatif, ce qui signifie que l'état stationnaire est un point-selle. Si $\sigma<s$, la stabilité dépend de la valeur de $D$. A partir de l'équation (20), on peut remarquer que $D$ décroît avec $\bar{\varepsilon}_{\lambda}$, de $+\infty$ lorsque $\bar{\varepsilon}_{\lambda}$ tend vers 0 , vers $D=1 /(1-s)-\delta$ lorsque $\bar{\varepsilon}_{\lambda}$ tend vers $+\infty$. Comme $1 /(1-s)-\delta \in(0,1)$ d'après l'Hypothèse 3 , nous en déduisons que $D \in(0,1)$ lorsque $\bar{\varepsilon}_{\lambda}>\bar{\varepsilon}_{\lambda_{H}}, D=1$ lorsque $\bar{\varepsilon}_{\lambda}=\bar{\varepsilon}_{\lambda_{H}}$ et $D>1$ lorsque $\bar{\varepsilon}_{\lambda}<\bar{\varepsilon}_{\lambda_{H}}$. Le point (ii) de la proposition en découle.

Cette proposition montre donc que l'apparition de fluctuations dues à la volatilité des anticipations (indétermination de l'équilibre), et l'existence de cycles déterministes (bifurcation de Hopf) requièrent une substitution entre le capital et le travail suffisamment faible et une moyennne des élasticités des offres de travail suffisamment élevée.

La question qui nous intéresse maintenant est de savoir quel effet va avoir une augmentation de l'hétérogénéité entre les agents à travers une plus grande dispersion des élasticités $\varepsilon_{\lambda_{i}}$. Nous avons défini un accroissement de l'hétérogénéité comme étant une augmentation de la dispersion à moyenne $\bar{\varepsilon}_{\lambda}$ constante. La simple observation des équations (19) et (20) ainsi que des résultats énoncés dans la Proposition 3, nous conduit à la conclusion suivante :

PROPOSITION 4. Toute augmentation ou réduction de l'hétérogénéité, à travers une variation de la dispersion des élasticités $\varepsilon_{\lambda_{i}}$ à moyenne $\bar{\varepsilon}_{\lambda}$ constante, n'affecte pas la stabilité locale de l'état stationnaire.

Ce résultat provient du fait que seule la moyenne des élasticités $\varepsilon_{\lambda_{i}}$ (moment d'ordre un) affecte la dynamique locale, alors qu'aucun moment d'ordre deux, comme la variance, n'influence la matrice Jacobienne. Une plus grande hétérogénéité entre les consommateurs ne stabilise ni ne déstabilise l'économie, puisqu'elle n'altère pas les conditions pour l'apparition de fluctuations dues à la volatilité des anticipations des agents. Dans ce modèle, l'agent représentatif semble donc être une représentation satisfaisante du comportement d'une population hétérogène de consommateurs, bien qu'au contraire du modèle à agent à durée de vie infinie, les théorèmes du bien-être ne s'appliquent pas.

De manière à donner plus de signification à notre résultat d'indétermination de l'équilibre, nous allons lui fournir une interprétation en termes de fluctuations dues aux anticipations auto-réalisatrices des consommateurs. Supposons que les agents coordonnent leurs anticipations sur une hausse du taux d'intérêt réel futur. D'après l'équation (8), chacun d'entre eux va accroître son offre de travail. Agrégeant ces comportements, l'offre de travail moyenne va augmenter. Celle-ci 
a un effet négatif sur le revenu salarial $w l$ et donc sur l'accumulation de capital si et seulement si $1-s / \sigma<0 .{ }^{10}$ Dans ce cas, le taux d'intérêt futur va effectivement augmenter et les anticipations des agents sont bien auto-réalisatrices. Que peuton dire maintenant à propos du rôle de l'hétérogénéité ? En prenant la moyenne des comportements des consommateurs comme fixe, l'hétérogénéité n'influence pas la dynamique, parce que l'accumulation de capital dépend des offres de travail individuelles uniquement à travers l'offre de travail moyenne (équation (9)), et non sous une autre forme.

Finalement, il est intéressant de noter que le résultat obtenu dans cette section (Proposition 4) peut être étendu au cas où la production bénéficie d'externalités dans la production, croissante avec le capital et le travail agrégés. En effet, l'introduction d'une telle imperfection de marché modifie uniquement le secteur de la production sans changer le comportement des consommateurs.

\section{RÉFÉRENCES BIBLIOGRAPHIQUES}

BECKER R. et FOIAS C. [1987], «A Characterization of Ramsey Equilibrium», Journal of Economic Theory, 41, p. 173-184.

BECKER R. et FOIAS C. [1994], «The Local Bifurcation of Ramsey Equilibrium», Economic Theory, 4, p. 719-744.

BENHABIB J. et FARMER R. [1999], «Indeterminacy and Sunspots in Macroeconomics», dans Taylor J.B. et Woodford M. (eds), Handbook of Macroeconomics, North-Holland, Amsterdam, p. 387-448.

CAZZAVILlAN G., LLOYD-BRAGA T. et PINTUS P. [1998], «Multiple Steady States and Endogenous Fluctuations with Increasing Returns to Scale in Production», Journal of Economic Theory, 80, p. 60-107.

GUESNERIE R. et WOODFORD M. [1992], «Endogenous Fluctuations», dans Laffont J.J. (ed), Advances in Economic Theory: Proceedings of the $6^{\text {th }}$ World Congress of the Econometric Society, Cambridge University Press, Cambridge (MA), vol. 6, p. 289-412.

GHIGLINO C. et OLSZAK-DUQUENNE M. [2005], «On the Impact of Heterogeneity on Indeterminacy», International Economic Review, 46, p. 171188.

GHIGLINO C. et SORGER G. [2002], «Poverty Traps, Indeterminacy, and the Wealth Distribution», Journal of Economic Theory, 105, p. 120-139.

REICHLIN P. [1986], «Equilibrium Cycles in an Overlapping Generations Economy with Production», Journal of Economic Theory, 40, p. 89-103.

WOODFORD M. [1986], «Stationary Sunspot Equilibria in a Finance Constrained Economy», Journal of Economic Theory, 40, p. 128-137.

\footnotetext{
${ }^{10}$ Cette dernière inégalité est obtenue en déterminant l'élasticité de $w l$ par rapport à $l$ et en utilisant l'équation (3).
} 